\title{
Message Complexity Analysis of MANET Address Autoconfiguration Algorithms in Group Merging Case
}

\author{
Sang-Chul Kim \\ School of Computer Science, Kookmin University, \\ 861-1, Chongnung-dong, Songbuk-gu, Seoul, 136-702 Korea \\ sckim7@kookmin.ac.kr
}

\begin{abstract}
This paper focuses on the derivation of the message complexity when two mobile ad hoc network (MANET) groups merge together, where the network groups already have been configured with IP addresses by using address autoconfiguration protocols (AAPs). The message complexity of the MANET group merging case (GMC) in Strong DAD, Weak DAD with proactive routing protocols (WDP), Weak DAD with on-demand routing protocols (WDO), and MANETconf has been derived respectively. In order to verify the derived bounds, analytical simulations that quantify the message complexity of the address autoconfiguration process based on the different conflict probabilities are conducted.
\end{abstract}

Keywords: Mobile Ad hoc Networks, Group Merge, Address Autoconfiguration, Message Complexity.

\section{Introduction}

Clustering (or grouping) of mobile nodes provides effective and efficient means to control routing and addressing in MANETs. In MANET, as the network grows (including more nodes) if hierarchical routing schemes are applied the control overhead is known to increase in a scalable fashion compared to using flat routing techniques. Due to this reason, several hierarchical routing protocols have been developed to enable scalable MANET routing solutions [1]. Due to the lack of any centralized control and possible node mobility in MANETs, many issues at the network, medium access, and physical layers currently remain as research topics since no counterparts in the wired networks or cellular networks can satisfy MANET requirements. One of the main criteria in determining efficiency in MANETs is the scalability of the control signaling. This issue becomes even more serious when MANET groups merge, as the addressing and new route establishment is required for multiple nodes simultaneously $[2,3,4,5]$. In mobile IPv6 networks, a mobile node can select its own IP address (using the subnet's prefix) but needs to obtain confirmation from the subnetwork before being permitted to use the chosen address. The confirmation process is based on a duplicated address detection (DAD) operation. The DAD operation is one of the 
most important processes of address autoconfiguration. Currently, Weak DAD, Strong DAD, and MANETconf have been proposed as candidate algorithms for DAD address autoconfiguration $[6,7]$. The broadcast storm problem introduced in [8] is a serious problem in MANET operations, and hence several algorithms are introduced in [9] to reduce the number of broadcast messages. The authors of [9] conclude that finding a minimum flood tree which gives the minimum number of forward nodes is proven to be a NP-complete problem, where the minimum flood tree is derived in [10]. In order to provide scalability, adaptability, and autonomy, Shen proposes the Cluster-based Topology Control (CLTC) algorithm [11] that uses a clustering strategy as well as a topology control algorithm with help of changing the transmission power. Shen uses the message complexity to statistically measure the performance of the CLTC protocol. The authors in [1] calculate the storage complexity and communication complexity to analyze the scalability of various MANET routing protocols and introduce the routing overhead of periodically updated Link State (LS) messages, which is known to follow the order of $O\left(N^{2}\right)$, where $N$ indicates the number of nodes in a MANET. However, a message complexity analysis and comparison among the IP address autoconfiguration protocols for MANET GMC has not been conducted yet. Therefore, in this paper, the upper bounds of the message complexity of the IP address autoconfiguration protocols for MANETs are derived for the GMC. In order to verify the derived bounds, analytical simulations that quantify the message complexity of the address autoconfiguration process based on the different conflict probabilities are conducted. In addition, the acronyms of messages and nomenclatures of the retry count variables used in this paper are summarized in Table 1.

Table 1. Acronym table [*: variable]

\begin{tabular}{|c|c||c|c||c|c|}
\hline Acronym & Message & Acronym & Message & Acronym & Message \\
\hline \hline$A B$ & Abort & $A P$ & Address Reply & $N Q$ & Neighbor Query \\
\hline$A C$ & Address Cleanup & $A Q$ & Address Request & $R R$ & Route Reply \\
\hline$A D$ & Advertised & $I R$ & Initiator Reply & $R Q$ & Route Request \\
\hline$A E$ & Address Error & $I Q$ & Initiator Request & $R T$ & Requester Request \\
\hline$A L$ & Allocated & $L S$ & Link State & $m$ & DAD retry count limit* \\
\hline$A O$ & Allocation & $N R$ & Neighbor Reply & $n$ & retry count limit* \\
\hline
\end{tabular}

\section{Message Complexity Analysis}

A MANET can be represented as an undirected graph $G(\boldsymbol{V}, \boldsymbol{E})$ where $\boldsymbol{V}$ is a finite nonempty set of nodes, which can be represented as $\boldsymbol{V}=\left\{V_{1}^{G}, V_{2}^{G}, \cdots, V_{W}^{G}\right\}$ where $|\boldsymbol{V}|=W$ and $\boldsymbol{E}$ is a collection of pairs of distinct nodes from $\boldsymbol{V}$ that form a link, which can be represented as $\boldsymbol{E}=\left\{E_{1}^{G}, E_{2}^{G}, \cdots, E_{W}^{G}\right\}$ [12]. A connected, acyclic, undirected graph which contains all nodes is defined as a free tree. $\boldsymbol{V}$ can be partitioned into several subgraphs $\boldsymbol{V}_{1}, \boldsymbol{V}_{2}, \cdots, \boldsymbol{V}_{k}, \cdots, \boldsymbol{V}_{n}$ where each 
partition subgraph is called as a free tree and $\left|\boldsymbol{V}_{1}\right|+\left|\boldsymbol{V}_{2}\right|+\cdots+\left|\boldsymbol{V}_{n}\right|=W$. A partitioned subgraph $\boldsymbol{V}_{k}$ is represented as a free tree $P(V, E)$, in which a node set $V$ is represented as $V_{1}, V_{2}, \cdots, V_{N}$ and $|V|$ equals $N$ containing all nodes in the partitioned subgraph $\boldsymbol{V}_{k}$, where $N \leq W$.

In this paper, the most common flooding method is used to broadcast an $A Q$ message where every node retransmits an $A Q$ message to its entire one-hop neighbors whenever it receives the first copy of the $A Q$ message. Since each member node in a free tree will relay the $A Q$ message initiated at node $\boldsymbol{V}_{i}$, the maximum number of nodes relaying an $A Q$ message is $N-1$, where the rule of discarding duplicated messages at a node is adopted. Therefore, the maximum number of $A Q$ messages broadcasted or relayed in the free tree is $N$, which can be represented as $O(N)$. The variable $t$ is defined as the largest number of nodes in a communication path based on the routing tree, including the source node.

Definition 1. For a MANET routing tree with $t$ nodes in the maximum length path, $O(t)$ is the upper bound of the maximum number of unicasted or relayed AP messages when a node unicasts an AP message.

In order to analyze the GMC, a scenario is considered where two MANET groups $\boldsymbol{V}_{i}$ and $\boldsymbol{V}_{j}$, where $\left|\boldsymbol{V}_{i}\right|=N_{1},\left|\boldsymbol{V}_{j}\right|=N_{2}$, and $N_{1} \leq N_{2}$, merge into each other and a node in the $\boldsymbol{V}_{i}$ finds an IP address that is duplicated in the $\boldsymbol{V}_{j}$ based on a routing message such as Hello received (Strong DAD), or based on the $L S$, $R Q$, or $R R$ message received (Weak DAD). It is assumed that in order to include the fields of Partition Identity $(P I)$ and the number of nodes $\left(N_{1}\right.$ or $\left.N_{2}\right)$, the routing message is modified in each group for Strong DAD, or $L S, R Q$, or $R R$ message is modified for Weak DAD. All nodes of a group know their group's PI (Lowest IP, UUID), where the Universal Unique ID (UUID) is the MAC address of the lowest IP address node. When the two nodes $I$ and $J$ associated in two different groups become neighboring nodes to each other, two nodes $I$ and $J$ detect the merger of two different MANET groups with help of the routing message or $L S, R Q$, or $R R$ message.

In order to analyze the GMC with Strong DAD, since the message complexity of the $\boldsymbol{V}_{j}$ is defined as $n\left(m O\left(N_{1}+N_{2}\right)+O(t)\right)$ as shown in Fig. 1(a), in the worst case, $N_{1}$ nodes in the $\boldsymbol{V}_{i}$ need to verify their IP addresses in a merged MANET. The message complexity of the GMC can be represented as $n N_{1}\left(m O\left(N_{1}+N_{2}\right)+O(t)\right)$, where each node in the $\boldsymbol{V}_{i}$ generates the message complexity of $n\left(m O\left(N_{1}+N_{2}\right)+O(t)\right)$, which concludes the following corollary.

Corollary 1. $n N_{1}\left(m O\left(N_{1}+N_{2}\right)+O(t)\right)$ is the upper bound of the maximum number of broadcasted/relayed $A Q$ messages and unicasted/relayed $A P$ messages of the GMC with Strong DAD.

Since the message complexity in the $\boldsymbol{V}_{i}$ and $\boldsymbol{V}_{j}$ caused by a node in the $\boldsymbol{V}_{i}$ has been defined as $n\left(O\left(N_{1}+N_{2}\right)+O(t)\right)$ and $n\left(O\left(N_{1}+N_{2}\right)+2 O(t)\right)$ as shown in Fig. 1(b), in the worst case, $N_{1}$ nodes in the $\boldsymbol{V}_{i}$ need to verify their IP addresses in a merged MANET. Therefore, the message complexity of the GMC can be represented as $N_{1}\left(n\left(O\left(N_{1}+N_{2}\right)+O(t)\right)\right)$ in WDP and $N_{1}\left(n\left(O\left(N_{1}+N_{2}\right)+2 O(t)\right)\right)$ in 


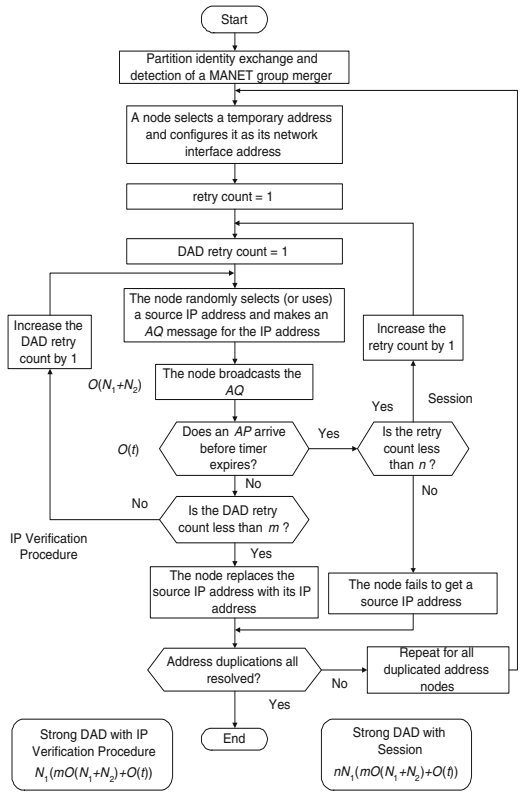

(a) Strong DAD

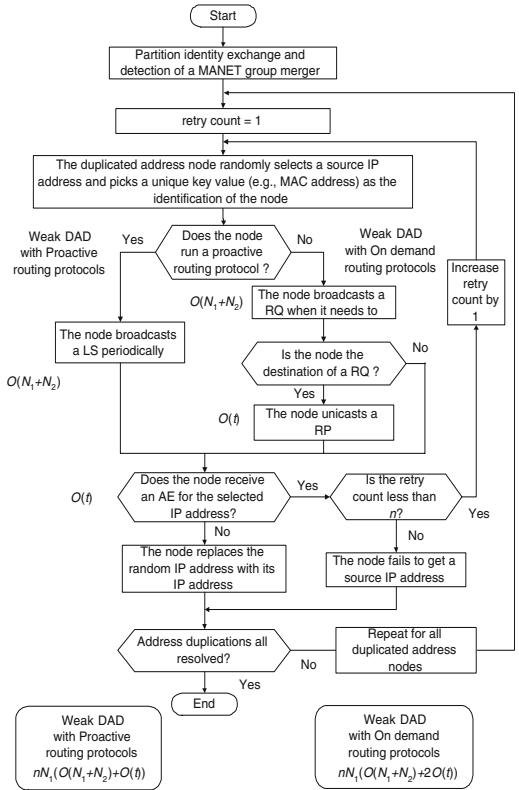

(b) WDP and WDO

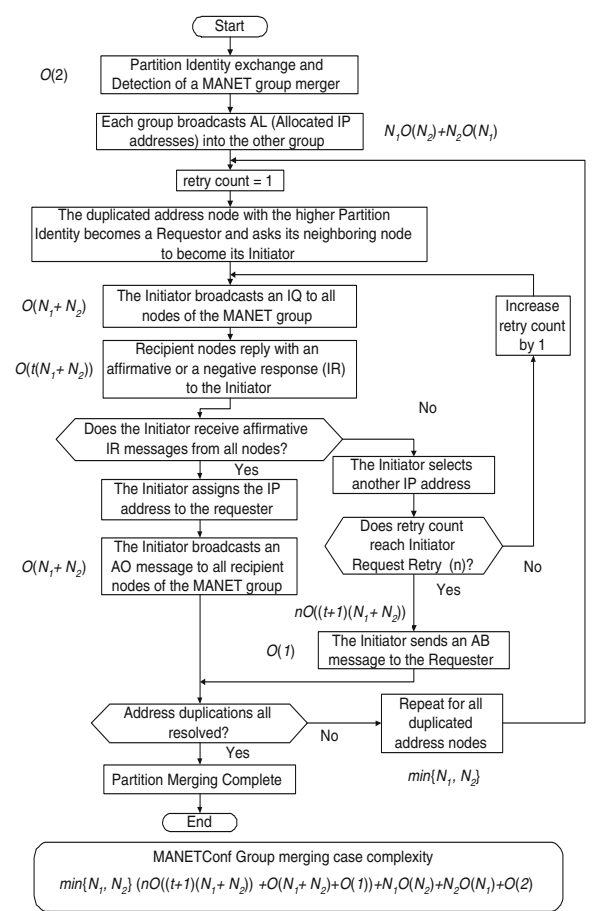

(c) MANETconf

Fig. 1. The flowcharts of Strong DAD, Weak DAD, and MANETconf operations 
WDO where each node in the $\boldsymbol{V}_{i}$ generates the message complexity of $n\left(O\left(N_{1}+\right.\right.$ $\left.\left.N_{2}\right)+O(t)\right)$ in WDP and $n\left(O\left(N_{1}+N_{2}\right)+2 O(t)\right)$ in WDO. Based on the results, the following corollary can be derived.

Corollary 2. $n N_{1}\left(O\left(N_{1}+N_{2}\right)+O(t)\right)$ (WDP) and $n N_{1}\left(O\left(N_{1}+N_{2}\right)+2 O(t)\right)$ (WDO) are the upper bounds of the maximum number of broadcasted/relayed $L S$ messages and unicasted/relayed AE messages of the GMC using WDP.

In the GMC of MANETconf, based on the flowchart as shown in Fig. 1(c), when the two nodes $I$ and $J$ associated in two different groups, which are $\boldsymbol{V}_{i}$ and $\boldsymbol{V}_{j}$ respectively, become neighboring nodes to each other, they exchange their Partition Identities. The nodes $I$ and $J$ can detect the merger of two different groups when the two nodes ( $I$ and $J$ of each group) exchange their $A L$ sets of IP addresses, which must contain the group's $P I$. Since the $A L$ message is composed of a list of IP addresses of a group, the size of $A L$ message could be larger than the maximum transfer unit (MTU) permitted in the MANET. It is assumed that in the worst case, the required MTU is small where each $A L$ message packet contains only a single IP address (and overhead of the upper layers). Therefore, in the upper bound case, an $A L$ message from $\boldsymbol{V}_{j}$ is segmented into $N_{2}$ number of MTU sized messages and transmitted in $\boldsymbol{V}_{i}$. In addition, the $A L$ message from $\boldsymbol{V}_{j}$ is segmented into $N_{1}$ number of MTU sized messages and transmitted in $\boldsymbol{V}_{j}$. The algorithm requires all nodes in $\boldsymbol{V}_{i}$ to broadcast the $A L$ messages transferred from $\boldsymbol{V}_{j}$, and all nodes in $\boldsymbol{V}_{i}$ have to broadcast $N_{2}$ number of $A L$ messages. As a result, the message complexity can be represented as $N_{2} O\left(N_{1}\right)$. Likewise, all nodes in $\boldsymbol{V}_{j}$ need to broadcast the $A L$ messages transferred from $\boldsymbol{V}_{i}$, and therefore, all nodes in the $\boldsymbol{V}_{j}$ have to broadcast $N_{1}$ number of $A L$ messages. As a result, the message complexity can be represented as $N_{1} O\left(N_{2}\right)$. Therefore, the message complexity due to broadcasting the $A L$ messages in $\boldsymbol{V}_{i}$ and $\boldsymbol{V}_{j}$ can be represented as $N_{1} O\left(N_{2}\right)+N_{2} O\left(N_{1}\right)$. The duplicated address node with the higher $P I$ will become the Requestor asking its neighboring node to become its Initiator. Among the duplicated addresses nodes, the node of the group that has the higher PI (i.e., comparing the lowest IP address of each group first, and if needed, also by comparing the UUID of each group) will become the Requestor which chooses one of its neighbors with a non-conflicting address as its Initiator to send an $I Q$ message. Any nodes detecting conflicted IP addresses become Initiators, where each Initiator broadcasts an $I Q$ message to all nodes of the group with the address of the Requester. The message complexity upper bound of broadcasting $I Q$ messages can be represented as $O\left(N_{1}+N_{2}\right)$ since the $I Q$ message is broadcasted into the merged MANET. Recipient nodes will reply with an affirmative or a negative response (using $I R$ message) to the Initiator. Therefore, the message complexity upper bound of unicasting IR messages can be represented as $O\left(t\left(N_{1}+N_{2}\right)\right)$ since all nodes $\left(N_{1}+N_{2}\right)$ unicast the $I R$ message and each $I R$ message has the message complexity upper bound of $O(t)$ based on Definition 1. If the initiator receives positive $I R$ messages from all recipient nodes, it broadcasts an $A O$ message to all recipient nodes of the group. The message complexity upper bound of broadcasting the $A O$ message can be represented as $O\left(N_{1}+N_{2}\right)$. Therefore, the following corollary can be derived. 
Corollary 3. In an IP verification procedure of the GMC, $O\left((t+1)\left(N_{1}+N_{2}\right)\right)$ is the upper bound of the maximum number of broadcasted or relayed $I Q$ messages and unicasted or relayed IR messages when a node needs to verify its IP address in a MANET.

If the initiator receives any negative $I R$ messages from its recipient nodes, it selects another IP address and repeats the steps of broadcasting $I Q$ and receiving $I R$ messages until the retry count reaches the retry count limit $(n)$. Therefore, the message complexity of broadcasting $A O$ messages and receiving $I R$ messages until the retry count is less than $n$ can be represented as $n\left(O\left(N_{1}+N_{2}\right)+O\left(t\left(N_{1}+\right.\right.\right.$ $\left.N_{2}\right)$ )). After $n$ times of repetition, if the initiator receives negative $I R$ messages, it sends an $A B$ message to the requestor. The message complexity of unicasting the $A B$ message can be represented as $O(1)$. Based on the above results, the following corollary can be derived.

Corollary 4. In a session of a GMC, $n O\left((t+1)\left(N_{1}+N_{2}\right)\right)+O\left(N_{1}+N_{2}\right)+O(1)$ $i s$ the upper bound of the maximum number of broadcasted or relayed IQ and $A O$ messages and unicasted or relayed IR and AB messages in MANETconf.

The above session procedure per a duplicated address node should be repeated until all duplicated address nodes are resolved. The repetition number of the session procedure in a merged MANET is $\min \left(N_{1}, N_{2}\right)$. Based on the above results, the following corollary can be derived.

Corollary 5. In resolving all duplicated addresses of a GMC with MANETconf, $\min \left(N_{1}, N_{2}\right)\left\{n O\left((t+1)\left(N_{1}+N_{2}\right)\right)+O\left(N_{1}+N_{2}\right)+O(1)\right\}+O(2)+N_{1} O\left(N_{2}\right)+$ $\mathrm{N}_{2} \mathrm{O}\left(\mathrm{N}_{1}\right)$ is the upper bound of the maximum number of messages.

\section{$3 \quad$ Numerical Results}

In order to analyze the message complexity of each AAP, a computer simulator was developed where nodes are randomly distributed with uniform density in a network area of $1 \mathrm{~km}^{2}$. A discrete-event simulator was developed in Matlab in order to verify the various network topologies and to calculate the message complexity of each AAP. The random node generator and simulator performance was verified (for the numbers of nodes 100, 125, 150, and 175) so that the average number of nodes per cluster as well as several specs in the adaptive dynamic backbone (ADB) algorithm [11] matched with the results in [11], which was performed by QualNet, with less than a $1 \%$ difference for almost all cases. In our analysis, the conflict probability is defined as the probability in which the IP address that a node requests to use is already in use in the group. Dijkstra's shortest path algorithm at each node is used to calculate the number of hops in unicasting or relaying an unicasted $A P$ message from a destination node to a source node. In the Strong DAD, five is used for retry count limit $(n)$ and three is used for DAD retry count limit $(m)$. In the Weak DAD and MANETconf, five is used for retry count limit $(n)$ and one is used for DAD retry count limit $(m)$. 
In addition, $100 \mathrm{~m}$ is selected as the transmission range of nodes. The number of nodes is varied from 10 to 50. In Fig. 2, it can be observed that at the conflict probability of 0.5 and 0.7 , WDP has the smallest message complexity and Strong $\mathrm{DAD}$ has the largest message complexity. In the range of 10 to 35 nodes at the conflict probability of 0.9 , and also for the case of 10 and 25 nodes at the conflict probability of 1 , WDP has the smallest message complexity and Strong DAD has the largest message complexity in the GMC. In addition, it can be calculated that with the increase of the conflict probability from 0.5 to 1 , the maximum overhead percentage of the message complexity of WDO increases gradually from $28.41 \%$ to $35.96 \%$ and then decreases to $33.52 \%$, the maximum overhead percentage of the message complexity of MANETconf is decreased from $318.10 \%$ to $232.48 \%$ gradually, and the maximum overhead percentage of the message complexity of Strong DAD decreases rapidly from $408.38 \%$ to $172.29 \%$.

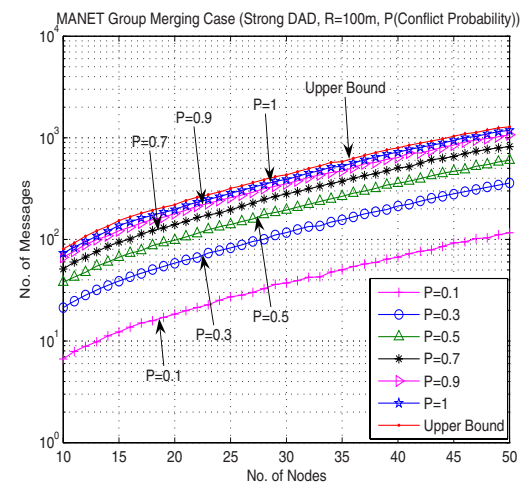

(a) Strong DAD

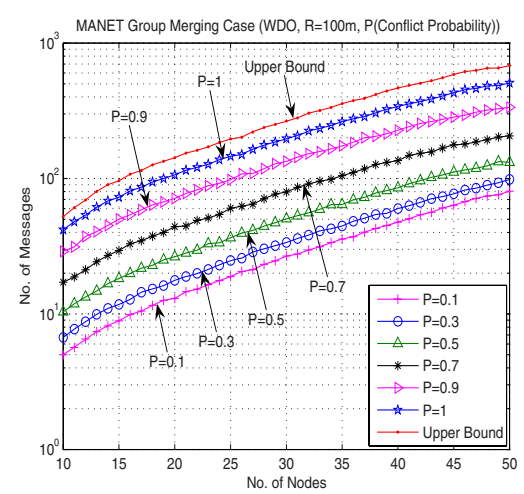

(c) WDO

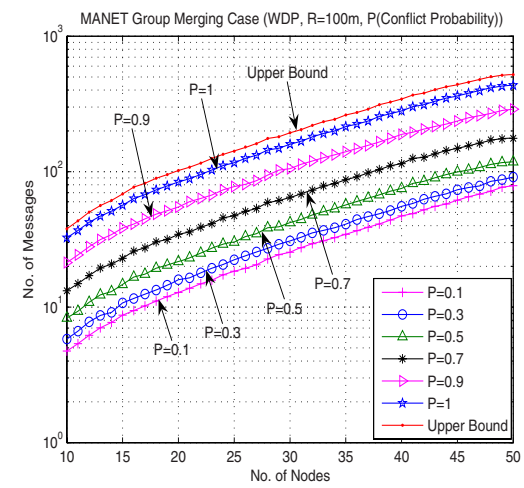

(b) WDP

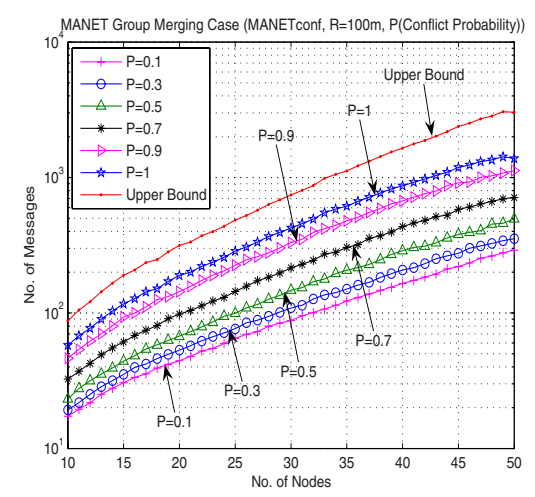

(d) MANETconf

Fig. 2. Message complexities of Strong DAD, WDP, WDO, and MANETconf 


\section{Conclusion}

The main objective of this paper is to propose a novel method to perform a quantitative analysis of message complexity and to compare the message complexity among the MANET AAPs in the GMC. To conduct a quantitative analysis of message complexity, the analysis of the worst case scenario is conducted in this paper. By introducing the retry count limit $(n)$ of a session in Strong DAD, the possibility of resulting in an infinite loop has been removed. By adapting the mechanism of the replying $A E$ message, the Weak DAD is equipped to properly react when solving duplicated IP address situations. Based on the simulation results, when nominal $n, m, t, N$ values and transmission range have been assigned with $p=0.5$ and 0.7 , the message complexity can be compared as follows: WDP $<$ WDO $<$ MANETconf $<$ Strong DAD. However, for the case where the conflict probability is 0.9 or 1 , the message complexity of the MANET GMC can be compared as follows: WDP $<$ WDO $<$ Strong DAD $<$ MANETconf. The results of this paper provide a direct comparison of scalability of DAD schemes based on MANET group merging cases. The methodology applied in this paper can be used to analyze newly developed DAD schemes in the future, which is one of the objectives that lead to conducting this research.

\section{References}

1. X. Hong, K. Xu, M. Gerla: Scalable Routing Protocol for Mobile Ad Hoc Networks. IEEE Network, pp.11-21 (2002)

2. C.-C. Chiang and M. Gerla: Routing in Clustered Multihop Mobile Wireless Networks. Proc. Information Networking (ICOIN11), 3B-1.1-3B-1.9 (1997)

3. G. Pei, M. Gerla, X. Hong, C. C, Chiang: A Wireless Hierarchical Routing Protocol with Group Mobility. Proc. IEEE WCNC '99, New Orleans, LA (1999)

4. Z. J. Haas, M R. Pearlman, Prince Samar: The Zone Routing Protocol (ZRP) for Ad Hoc Networks. Internet Draft (2002) http://www.ietf.org/proceedings/02nov/ I-D/draft-ietf-manet-zone-zrp-04.txt

5. M. Gerla, X. Hong, L. Ma, G. Pei: Landmark Routing Protocol (LANMAR) for Large Scale Ad Hoc Networks. Internet Draft (2002) http://www.ietf.org/ proceedings/01dec/I-D/draft-ietf-manet-lanmar-02.txt

6. N. H. Vaidya: Weak duplicate address detection in mobile ad hoc networks. Proc. ACM MobiHoc, Lausanne Switzerland(2002) 206-216

7. S. Nesargi, R. Prakash: MANETconf: Configuration of hosts in a mobile ad hoc network. Proc. IEEE Infocom 2002, New York (2002)

8. S. Ni, Y. Tseng, Y. Chen, J. Sheu: The Broadcast Strom Problem in a Mobile Ad Hoc Network. Proc. ACM MobiCom (1999)

9. W. Lou, J. Wu: On reducing broadcast redundancy in Ad hoc wireless networks. IEEE Trans. on Mobile Computing, Vol. 1, No. 2 (2002) 111-122

10. H. Lim, C. Kim: Flooding in wireless ad hoc networks. Computer Comm. J., vol. 24, no.3-4 (2001) 353-363

11. C-. C. Shen, C. Srisathapornphat, R. L. Z. Huang, C. Jaikaeo, E. L. Lloyd: CLTC: A cluseter-based topology control framework for ad hoc networks. IEEE Trans. Mobile Computing, vol. 3, no.1 (2004) 18-32

12. J. Gross, J. Yellen. Graph Theory and Its Applications. CRC Press (1998) 